\title{
Detection of ULF electromagnetic emissions as a precursor to an earthquake in China with an improved polarization analysis
}

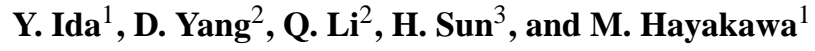 \\ ${ }^{1}$ Department of Electronic Engineering and Research Station on Seismo Electromagnetics, \\ The University of Electro-Communications, 1-5-1 Chofugaoka, Chofu, Tokyo, 182-8585, Japan \\ ${ }^{2}$ Institute of Geophysics, China Earthquake Administration, Beijing 100081, China \\ ${ }^{3}$ Kashi Observatory, Earthquake Administration of Xinjiang Uygur Autonomous Region, \\ Xinjiang 844000, China
}

Received: 16 May 2008 - Accepted: 1 July 2008 - Published: 30 July 2008

\begin{abstract}
An improved analysis of polarization (as the ratio of vertical magnetic field component to the horizontal one) has been developed, and applied to the approximately four years data (from 1 March 2003 to 31 December 2006) observed at Kashi station in China. It is concluded that the polarization ratio has exhibited an apparent increase only just before the earthquake on 1 September 2003 (magnitude $=6.1$ and epicentral distance of $116 \mathrm{~km}$ ).
\end{abstract}

\section{Introduction}

There have been recently accumulated a lot of evidences on electromagnetic emissions in a wide frequency range associated with earthquakes (EQs) (e.g., Hayakawa and Molchanov, 2002; Molchanov and Hayakawa, 2008). The lowest frequency range, ULF (ultra low frequency, with frequency less than $10 \mathrm{~Hz}$ ) is of practical importance in shortterm EQ prediction, because they are able to propagate easily up to the Earth's surface where a ULF sensor is installed.

The serious problem regarding these seismogenic ULF emissions is how to detect these weak signals. There have been developed different kinds of methods for the analysis; (1) polarization analysis by means of the ratio of vertical magnetic field component to the horizontal (Hayakawa et al., 1996), (2) fractal analysis (mono- and multi-) (Hayakawa et al., 1999; Gotoh et al., 2004; Smirnova et al., 2004; Ida and Hayakawa, 2006; Ida et al., 2005), (3) Principal component analysis (Gotoh et al., 2002) and singular value decomposition (Hattori et al., 2006), and so on.

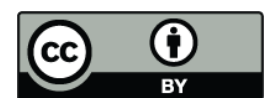

Correspondence to: M. Hayakawa hayakawa@whistler.ee.uec.ac.jp
In this paper we will use the polarization analysis as the simplest analysis method, but we have developed its improved one. This improved polarization method is applied to the ULF data observed in China during four years. We try to find out any significant precursory effect for two EQs in China near the observing situation of Kashi in Fig. 1. Finally, the results obtained in this paper would be compared with earlier results for the 1993 Guam EQ (Hayakawa et al., 1996, 1999) and Kagoshima EQs in 1999 (Hattori et al., 2006).

\section{ULF geomagnetic data and EQs}

ULF geomagnetic data are obtained at an observatory named Kashi (geographic coordinates; $39.5^{\circ} \mathrm{N}, 76.0^{\circ} \mathrm{E}$ ) as in Fig. 1. At this field site we observe three geomagnetic components ( $H: N S$ component, $D: E W$ component, and $Z$ :vertical component) by means of fluxgate sensors. The sampling frequency is $1 \mathrm{~Hz}$. Nearly four years data are utilized for the analysis: 1 March 2003 through 31 December 2006.

There were observed two rather big EQs (with magnitude greater than 6.0) near the ULF station of Kashi; an EQ on 1 September 2006 and another EQ on 25 February 2005. The magnitude of the former one is $M=6.1$ and that of the latter one is $M=6.0$. The distance of the former EQ with respect to the observatory is about $116 \mathrm{~km}$, while the epicenter distance for the next EQ is about $\sim 300 \mathrm{~km}$.

\section{Polarization analysis}

Polarization method as developed by Hayakawa et al. (1996) is based on the measurement of the ratio of spectral power of the vertical magnetic field $(Z)$ to the horizontal magnetic 


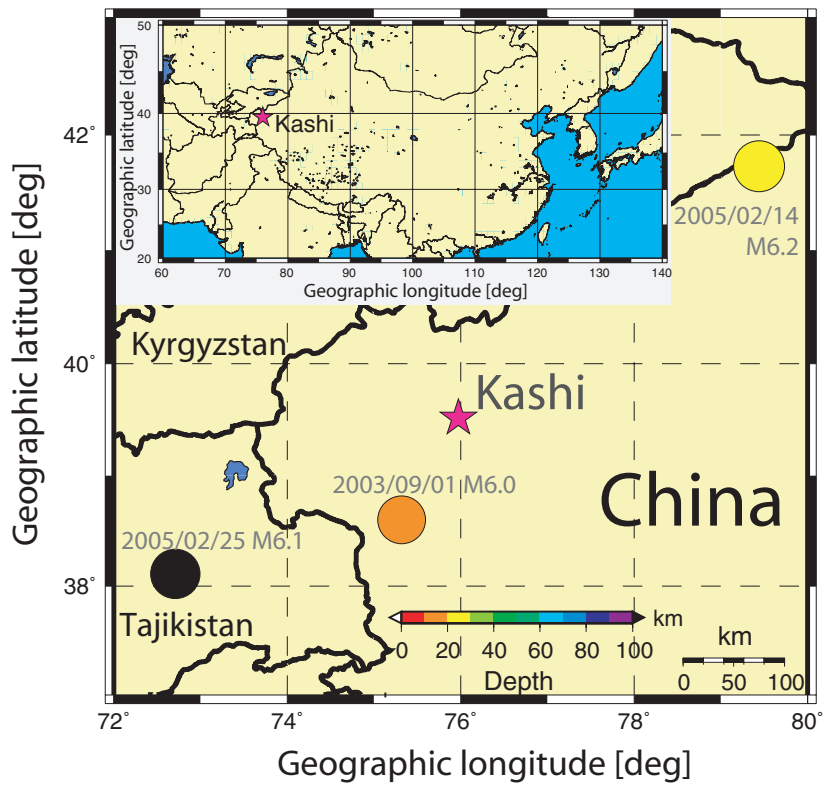

Fig. 1. The relative location of a ULF geomagnetic observatory, Kashi and two EQs (indicated by circles) occurred during the period of March 2003 to December 2006.

fields $(H$ and $D$ ) (i.e., $Z / H$ or $Z / D)$. This ratio is known to provide us with a lot of information whether the observed variation is of ionospheric orgin (or solar-terrestorial effect) or seismic-related. Generally speaking, the polarization ratio becomes generally larger when we have seismogenic emissions, while the geomagnetic variation is found to have smaller values (Hayakawa et al., 1996).

The time series ULF data are subject to the following signal processing. The use of FFT enables us to change the time series information into the frequency domain. The window size used is 1024, and the window function is Hamming. The analysis window is shifted without any overlapping. Secondly, the data at the frequency around $0.01 \mathrm{~Hz}(10 \mathrm{mHz})$ is picked up from the FFT result. This frequency is already known to represent seismogenic ULF emissions (Hayakawa et al., 2007), and this point was checked in this paper as well by changing the frequency in the analysis. The geomagnetic $D$ component at $\sim 0.01 \mathrm{~Hz}$ has a significant variation at the terminator time (Zomer et al., 2008), while geomagnetic $H$ and $Z$ components at day exhibit significant annual variations. Additionally, daytime geomagnetic data are likely to include more artificial noise than at night. So that, the average of each geomagnetic component $(H, D$ and $Z)$ is computed by using the data from U.T. $=18: 00$ to 21:00 (local nighttime) in order to reduce these effects. The values of $Z / H$ and $Z / D$ are computed as representing the daily data.

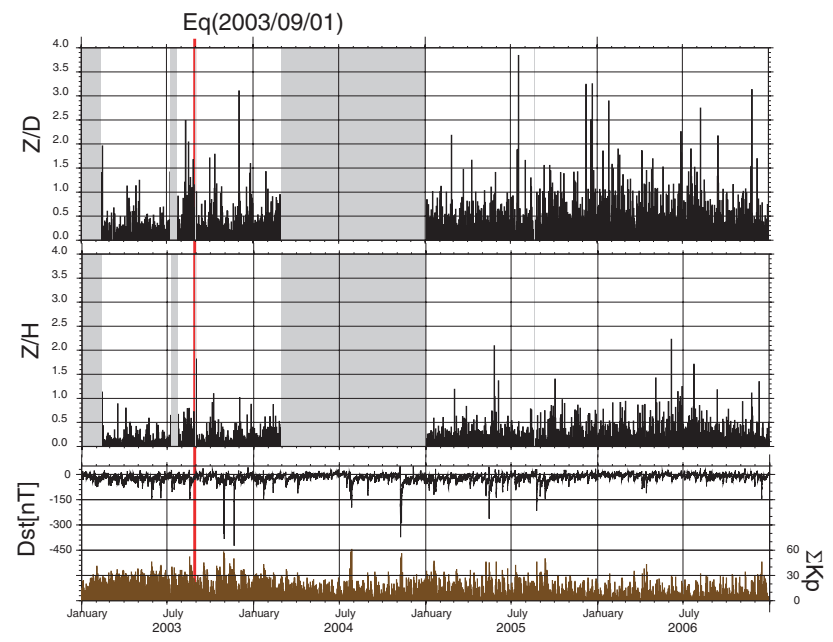

Fig. 2. Temporal polarization $(Z / D, Z / H)$ results (before standardization), Dst and $\Sigma \mathrm{Kp}$ indices. Eq is the EQ occurrence date.

\section{Observational results on polarization}

Figure 2 illustrates the temporal evolution of the polarization analysis $(Z / D$ and $Z / H)$ during the whole period from March 2003 to December 2006. The shaded areas indicate the periods of no observation due to some problems of the ULF system. A vertical line indicates the day of occurrence of the nearby EQ on 1 September 2003. The upper part of the bottom panel refers to the geomagnetic activity expressed by Dst [nT], while the lower part indicates the $\Sigma \mathrm{Kp}$ index. It is seen from this figure that a certain enhancement is seen in the $Z / D$ plot just before the EQ and also a less significant increase is seen in the $Z / H$ plot. However, we can notice so many other peaks in both plots of $Z / D$ and $H / D$. So that, we cannot say anything about the correlation between the polarization and an EQ. The two plots of $Z / D$ and $H / D$ are seen to have no correlation with either Dst or $\Sigma \mathrm{Kp}$. It is then likely that major causes for increasing the polarization during all the periods might be related to the different variability in each geomagnetic field component. Because the average and standard deviation of each field is very different.

So, we propose an improved polarization method in the following. Because of different behavior in each field component, we propose the standardization (normalization) of each geomagnetic field component as follows, which enables us to treat each field component equally. The standardized field component is defined by,

$E_{i}=\left(X_{i}-\mu_{i}\right) / \sigma_{i}$

where $X_{i}$ is the average value for one day mean for the $i$ component $(i=H, D$ and $Z), \mu_{i}$ is the average of the component $i$ over the whole period, and $\sigma_{i}$ is the standard deviation of the same component $i$ over the whole period. An increase 
in the polarization is resulted from not only an increase in $Z$, but also from a decrease in $H$ or $D$. So that, only when the standardized value of $H$ or $D$ component is exceeding 0.1 (that is, any significant change is observed), we compute the polarization and the corresponding result is given in Fig. 3.

It is seen that Fig. 3 is completely different from the result in Fig. 2. In this figure both values of $Z / D$ and $Z / H$ are found to exhibit a significant increase only before the $\mathrm{EQ}$, and no any significant changes are observed during the whole period. The indices of Dst and $\Sigma \mathrm{Kp}$ did not show any changes before the EQ, which means that the increase in polarization before the EQ has nothing to do with the geomagnetic activity, but it is closely associated the EQ.

As regards the second EQ on 25 February 2005, there seems to exhibit no any significant change. The epicentral distance for this EQ is about $300 \mathrm{~km}$, which is too far to detect any seismogenic effects (Hayakawa et al., 2007).

\section{Concluding remarks}

An improved polarization method developed in this paper is applied to the ULF data at Kashi station in China. Three geomagnetic field components behave in a different way, so that we have adopted general standardization (or normalization) by estimating the average and standard deviation during the whole period for each component. By using these standardized geomagnetic field quantities, it is found that the polarization as the ratio of vertical magnetic field component to that of horizontal magnetic field component $(Z / H, Z / D$ at a particular frequency of $\sim 0.01 \mathrm{~Hz}(10 \mathrm{mHz})$ exhibits a significant increase only before the EQ on 1 September 2003 (magnitude $=6.0$ and epicentral distance $=120 \mathrm{~km}$ ). We comment on the lead time for this EQ; that is, during 1 August to the end of August, we notice the enhancement in the polarization, with the maximum polarization value a few days before the EQ. This kind of ULF lead time seems to be consistent with earlier works by Hayakawa et al. (1996) and Hattori et al. (2006). No effect in ULF emissions is observed for the 2nd EQ on 25 February 2005, which can be easily understood in terms of a larger epicentral distance of $\sim 300 \mathrm{~km}$, being consistent with earlier statistics by Hayakawa et al. (2007).

Edited by: M. Contadakis

Reviewed by: two anonymous referees

\section{References}

Gotoh, K., Akinaga, Y., Hayakawa, M., and Hattori, K.: Principal component analysis of ULF geomagnetic data for Izu islands earthquakes in July 2000, J. Atmos. Electr., 22, 1-12, 2002.

Gotoh, K., Hayakawa, M., Smirnova, N. A., and Hattori, K.: Fractal analysis of seismogenic ULF emissions, Phys. Chem. Earth, 29, 419-424, 2004.

Hattori, K., Serita, A., Yoshino, C., Hayakawa, M., and Isezaki, N.: Singular spectral analysis and principal component analysis for signal discrimination of ULF geomagnetic data associated with

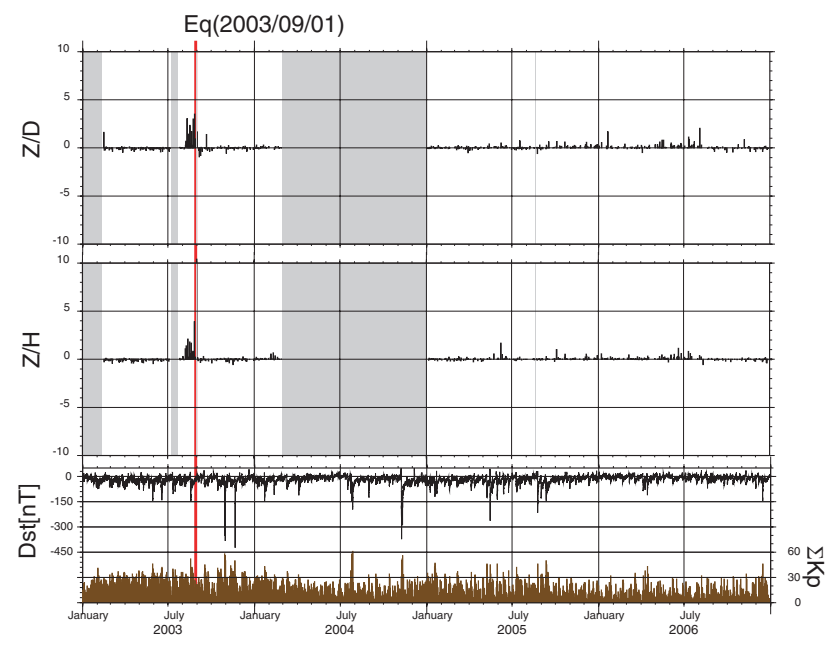

Fig. 3. Temporal evolution on the polarization $(Z / D, Z / H)$ results after standardization, Dst and $\Sigma \mathrm{Kp}$ indices. Eq is the EQ occurrence date.

2000 Izu Island earthquake swarm, Phys. Chem. Earth, 31, 281291, 2006.

Hayakawa, M., Kawate, R., Molchanov, O. A., and Yumoto, K.: Results of ultra-low-frequency magnetic field measurements during the Guam earthquake of 8 August 1993, Geophys. Res. Lett., 23, 241-244, 1996.

Hayakawa, M., Itoh, T., and Smirnova, N.: Fractal analysis of ULF geomagnetic data associated with the Guam earthquake on $8 \mathrm{Au}-$ gust 1993, Geophys. Res. Lett., 26(18), 2797-2800, 1999.

Hayakawa, M. and Molchanov, O. A.: Seismo Electromagnetics: Lithosphere - Atmosphere - Ionosphere Coupling, TERRAPUB, Tokyo, Japan, p. 477, 2002.

Hayakawa, M., Hattori, K., and Ohta, K.: Monitoring of ULF (ultralow-frequency) geomagnetic variations associated with earthquakes, Sensors, 7, 1108-1122, 2007.

Ida, Y., Hayakawa, M., Adalev, A., and Gotoh, K.: Multifractal analysis for the ULF geomagnetic data during the 1993 Guam earthquake, Nonlinear Proc. Geoph., 12, 157-162, 2005.

Ida, Y. and Hayakawa, M.: Fractal analysis for the ULF data during the 1993 Guam earthquake to study prefracture criticality, Nonlinear Proc. Geoph., 13, 409-412, 2006.

Molchanov, O. A. and Hayakawa, M.: Seismo-Electromagnetics and Related Phenomena, History and latest results, TERRAPUB, Tokyo, Japan, p. 189, 2008.

Smirnova, N., Hayakawa, M., and Gotoh, K.: Precursory behavior of fractal characteristics of the ULF electromagnetic fields in seismic active zones before strong earthquakes, Phys. Chem. Earth, 29, 445-451, 2004.

Zomer, A., Price, C., Alperovich, C., Finkelstein, M., and Merzer, M.: ULF amplitude observations at the dawn/dusk terminators, J. Atmos. Electr., 28, 21-29, 2008. 